\title{
Overload Induced Heat Shock Proteins (HSPs), MAPK and miRNA (miR-1 and miR133a) Response in Insulin-Resistant Skeletal Muscle
}

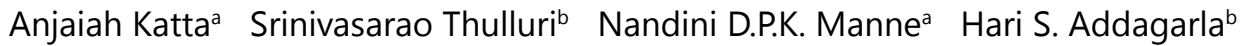 \\ Ravikumar Arvapalli ${ }^{b}$ Siva K. Nalabotu ${ }^{a}$ Muralikrishna Gadde ${ }^{b}$ Kevin M. Rice ${ }^{b}$ \\ Eric R. Blougha,b,c \\ aDepartment of Pharmacology, Physiology and Toxicology, Marshall University, Joan C. Edwards School \\ of Medicine, Huntington, WV; ${ }^{b} C e n t e r$ for Diagnostic Nanosystems, Marshall University, Huntington, \\ WV; ' Department of Pharmaceutical Sciences and Research, Marshall University, Huntington, WV
}

\section{Key Words}

Heat shock proteins $\cdot$ Mitogen-activated protein kinases $\cdot$ miRNA $\cdot$ Insulin resistance $\cdot$ Muscle overload

\begin{abstract}
Background: Insulin resistance (IR) may decrease muscle adaptability. Heat shock proteins (HSPs), mitogen-activated protein kinases (MAPKs), and miRNA are thought to play a role in muscle hypertrophy but it is unclear if IR may affect their regulation. Methods: Soleus muscles of lean Zucker (LZ) and insulin resistant obese Zucker (OZ) rats were overloaded for 7 or 21 days and subjected to immunoblotting and RT-PCR. Results: IR was associated with decreased muscle hypertrophy. Overload increased HSP27 phosphorylation in both the LZ and OZ rats at day 7 but only in the LZ at day 21. IR was associated with diminished overload induced MAPK phosphorylation and decreased expression of miR-1 and miR133. Overload decreased mir-1 levels in both the LZ and OZ but to a greater extent in the LZ animals. Conclusion: These results suggest that alterations in the regulation of HSPs, MAPKs and miRNA may be associated with the diminished hypertrophy of IR muscle.
\end{abstract}

Copyright $(2013$ S. Karger AG, Basel

\section{Introduction}

The adaptation of skeletal muscle to physiological and pathological stimuli is thought to be governed by the activation of intracellular signaling cascades. Previously we reported that insulin resistant muscle is characterized by a diminished hypertrophic response and that this 
response is associated with decreased activation of mammalian target of rapamycin (mTOR) signaling $[1,2]$. Whether other signaling systems may also play a role in this response is not well understood.

It is thought that the heat shock proteins (HSP) are important players in cellular adaptation and these proteins have been implicated in the chaperoning of unfolded proteins, the inhibition of apoptosis, and stabilization of the cytoskeleton [3-7]. Previous studies demonstrated the induction of HSP proteins following high force eccentric contraction, aerobic exercise, and during the development of muscle hypertrophy [8-10]; however it is not clear whether the regulation of these molecules is altered with insulin-resistance.

In addition to the Akt-mTOR-p70S6k signaling cascade [11-13] the mitogen-activated protein kinases (MAPKs) are another class of stress responsive proteins that are involved in the regulation of protein synthesis and cellular adaptation [14-16]. The MAPK proteins are activated by dual phosphorylation prior to their translocation to the nucleus, where they are thought to regulate various transcriptional factors $[17,18]$. Recent data has indicated that the extracellular regulated kinase $1 / 2$ (ERK1/2) and c-Jun N-terminal kinase (JNK) MAPK proteins play a crucial role in regulation of skeletal muscle hypertrophy following increased loading [19-23]. If or how insulin resistance may affect MAPK signaling in response to increased muscle loading has, to our knowledge, not been investigated.

In addition to these signaling cascades, other data has strongly suggested that musclespecific miRNAs (miR-1, miR-133a and miR-206) may be involved in the regulation of load induced skeletal muscle remodeling [24-26]. MicroRNAs are small endogenous 22-nucleotide, non-coding RNAs which repress gene expression by inhibiting mRNA translation by interacting with the 3'- untranslated region (3'UTR) of target mRNAs [2729]. Among the muscle-specific miRNA's, miR-1 and miR-133a are important for embryonic muscle growth [30], myoblast differentiation and proliferation as they regulate the expression of SRF, MyoD and MEF2 [31] while miR-206 plays a role in myogenesis [32]. Recent data from McCarthy and Esser [24], reported decreased expression of miR-1 and miR-133a following 7 days of functional overload and suggested this response acted to remove the repression of hypertrophic regulators [24]. How insulin resistance may affect the regulation of musclespecific miRNAs during muscle hypertrophy is currently unclear.

Utilizing the same animals and tissues detailed in our earlier work [1], here we investigate whether insulin resistance affects the regulation of the HSPs (HSP27, HSP60, HSP70 and HSP90), MAPKs (ERK1/2 and JNK) and muscle-specific miRNA's (miR-1 and miR133a), during muscle hypertrophy. Our data indicate that alterations in the regulation of HSPs, MAPKs and muscle-specific miRNAs regulation may be associated with the diminished ability of insulin resistant muscle to undergo hypertrophy following muscle overload.

\section{Materials and Methods}

Materials

Primary antibodies against HSP27 (\#2442), phospho-HSP27 (Ser82) (\#2401), HSP60 (\#4870), HSP70 (\#4872), HSP90 (\#4877), phospho-HSP90 (Thr5/7) (\#3488), p44/42 MAPK (ERK1/2) (\#9102), phospho-p44/42 MAPK (Thr202/Tyr204)(\#4377), JNK/SAPK (\#9252), phospho-JNK/SAPK (Thr183/ Tyr185) (\#9251), glyceraldehyde 3-phosphate dehydrogenase (GAPDH,\#2118) and secondary antibody conjugated with horseradish peroxidase (HRP) (anti-rabbit (\#7074) were purchased from Cell Signaling Technology (Beverly, MA). Laemmli 2 X sample buffer was purchased from Sigma-Aldrich (St. Louis, MO). Pierce Tissue Protein Extraction Reagent (T-PER), Pierce 660nm protein assay reagent (\#22660) and GE Healthcare Amersham ECL ${ }^{\mathrm{TM}}$ Western Blotting Detection Reagents (RPN2106) were obtained from Thermo Fisher Scientific Inc. (Rockford, IL). 10\% PAGEr Gold Precast gels were from procured from Lonza (Rockland, $\mathrm{ME})$. 


\section{Animal care and muscle overload}

All procedures were performed in accordance with the Marshall University Institutional Animal Care and Use Committee (IACUC) guidelines, using the criteria outlined by the American Association of Laboratory Animal Care (AALAC). The animals and tissues used in this study were from our earlier work [1]. Ten week old male LZ $(n=12)$ and $\mathrm{OZ}(\mathrm{n}=12)$ rats were purchased from the Charles River Laboratories and housed in pairs within an AAALAC approved vivarium. Animals were maintained at $22^{\circ} \pm 2{ }^{\circ} \mathrm{C}$ with a $12 \mathrm{H}: 12 \mathrm{H}$ dark-light cycle and acclimatized for two weeks prior to experimentation. Food and water were provided ad libitum.

Overload of the left soleus muscle was induced by surgical ablation of the proximal and medial twothirds of the lateral head of the gastrocnemius under ketamine-xylazine $(4: 1 ; 50 \mathrm{mg} / \mathrm{kg}, \mathrm{I} / \mathrm{P})$ anesthesia as previously described [33]. The contralateral right limb served as an intra-animal control and was subjected to the same surgical procedures however the gastrocnemius muscle was left intact. No signs of inactivity or infection were noted in any of the animals postoperatively.

Soleus muscles were collected (7 day $n=6$ LZ-7 and $n=6$ OZ-7, 21 day $n=6$ LZ-21 and $n=6$ 0Z-21) under ketamine-xylazine $(4: 1 ; 50 \mathrm{mg} / \mathrm{kg}, \mathrm{I} / \mathrm{P})$ anesthetic, carefully trimmed of any excess connective tissue, weighed and snap frozen in liquid nitrogen. Tissues were stored at $-80^{\circ} \mathrm{C}$ until further experimentation.

Tissue protein extraction

Pierce Tissue Protein Extraction Reagent (T-PER) $(10 \mathrm{~mL} / \mathrm{g}$ tissue; Rockford, IL, USA) containing phosphatase inhibitors (P5726, Sigma-Aldrich, Inc., St. Louis, MO, USA) and protease inhibitors (P8340, Sigma-Aldrich, Inc., St. Louis, MO, USA) was used for protein extraction as outlined by the manufacturer. After homogenization in ice cold TPER, extracts were incubated for $30 \mathrm{~min}$ on ice and then centrifuged at $12,000 \mathrm{x}$ g for $5 \mathrm{~min}$ at $4^{\circ} \mathrm{C}$. Protein concentrations were determined using the $660 \mathrm{~nm}$ protein assay (Thermo Scientific, Rockford, IL, USA). Samples were diluted with 2X Laemmli sample buffer (Sigma-Aldrich, Inc., St. Louis, MO, USA) and denatured for for 5 minutes at $95^{\circ} \mathrm{C}$ prior to SDS-PAGE.

\section{SDS-PAGE and immunoblotting}

Forty micrograms of total protein extract from each sample were separated using 10\% PAGEr Gold Precast gels (Lonza, Rockland, ME, USA) and then transferred to $0.45 \mu \mathrm{m}$ nitrocellulose membranes. Verification of transfer efficiency and equal loading of protein among lanes was determined by Ponceau S. Immunodetection of antigens was performed as described previously [34, 35]. In brief, membranes were blocked for $1 \mathrm{~h}$ at room temperature with constant shaking in blocking buffer ( $5 \%$ non-fat dry milk in TBS-T (20mM Tris-base, $150 \mathrm{mM} \mathrm{NaCl}, 0.05 \%$ Tween-20), $\mathrm{pH}$ 7.6) followed by serial washes in TBS-T at room temperature. Membranes were incubated overnight at $4{ }^{\circ} \mathrm{C}$ in primary antibody buffer (TBS-T containing $5 \%$ BSA, pH 7.6, 1:1000) and washed in TBS-T (3X 5 min each). After blocking for $1 \mathrm{~h}$ in TBS-T containing 5\% BSA, membranes were washed (TBS-T, $3 X 5 \mathrm{~min}$ ) and allowed to react with ECL reagent (Amersham ECL Western Blotting reagent) for 1 min to visualize protein bands. Quantification of target protein levels were performed using AlphaEaseFC image analysis software (Alpha Innotech, San Leandro, CA, USA). Bands of interest were normalized to glyceraldehyde 3-phosphate dehydrogenase (GAPDH).

\section{miRNA analysis}

Portions (25 mg) of each soleus muscle were pulverized with mortar and pestle in liquid nitrogen. miRNA was isolated using the miRVana miRNA Isolation Kit as outlined by the manufacturer (Ambion, Austin, TX). cDNA synthesis from miRNA was performed using the QuantiMir RT kit (System Bioscience) as detailed by the supplier. SYBR green-based real-time qPCR was performed by using a 7500 Real-Time PCR system (Applied Biosystems, Foster City, CA) and gene-specific primers for the miRNA of interest designed following the guidelines outlined within the QuantiMir RT kit. Melt analysis was used after each PCR run to ensure amplification of only a single product. To account for possible differences in the amount of starting miRNA, all samples were normalized to miR-206 as the expression of this molecule was not different between animal models or following overload. Relative fold changes in miRNA were determined from the $C_{t}$ values after normalization to mir-206 using the $2^{-\Delta c t}$ method (modified Levak method) [36]. 
A

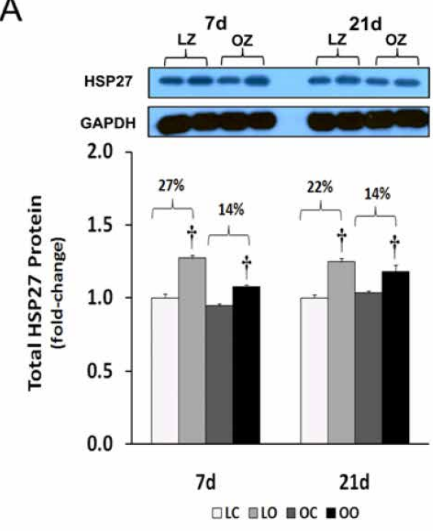

B

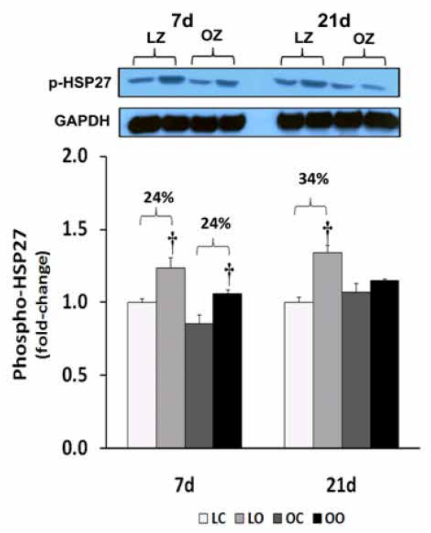

Fig. 1. Immunoblot analysis of the small heat shock protein, HSP27 (A) protein content and (B) phosphorylation at Ser82 in control and loaded soleus muscles of LZ and OZ. Protein quantification normalized to theabundance ofGAPDH protein; $n=6$. $†$ Significantlydifferent from contralateral control muscle. $P<0.05$.

\section{Statistical analysis}

Results are presented as mean \pm SE. Data were analyzed using the Sigma Stat 3.5 statistical program using two way analysis of variance (ANOVA) followed by the Student-Newman-Keuls post hoc testing where appropriate. Values of $P<0.05$ were considered to be statistically significant.

\section{Results}

The impaired hypertrophic response of insulin resistant skeletal muscle is associated with diminished HSP27 phosphorylation

As indicated previously, significant increase in body weight was higher in the OZ rats compared to the LZ rats at both 7 days $(464 \pm 12 \mathrm{gm} v$ s. $302 \pm 3 \mathrm{gm} ; P<0.05)$ and 21 days $(460 \pm 26$ vs. $289 \pm 10 \mathrm{gm} ; P<0.05)$ [1]. In contrast, the wet weights of soleus were found to be significantly lower in the OZ rats versus LZ rats at both 7 days $(128 \pm 11 \mathrm{mg} v$ s. $150 \pm 6$ mg; $P<0.05)$ and 21 days $(128 \pm 6.5 \mathrm{mg} v s .141 \pm 18.3 \mathrm{mg} ; P<0.05)$ of muscle overload. To examine the effect of insulin resistance on the regulation of HSP molecules with overload, we compared the expression and phosphorylation levels of HSP27, HSP60, HSP70 and HSP90. Muscle overload did not alter the expression of HSP60, HSP70 and HSP90 in either the LZ or OZ rats (Fig. 2 and 3A). The expression of the small heat shock protein HSP27 was increased in both lean and obese zucker animals after 7 and 21 days of overload $(P<0.05$; Fig. 1A). The phosphorylation of HSP27 at Ser 82 was increased in both LZ and OZ animals after 7 days of increased loading, while it was significantly increased only in the lean Zucker rats after 21 days of overload $(P<0.05$; Fig. 1B).

Insulin resistance alters the overload-induced phosphorylation of MAPK proteins in slow twitch soleus muscle

To investigate the effect of insulin resistance on the activation of MAPK proteins with overload, we compared the protein content and phosphorylation of p44/42 MAPK (ERK1/2) and JNK / SAPK between control and overloaded muscles. The amount of ERK1/2 was unaltered with muscle overload in both LZ and $\mathrm{OZ}$ animals, while the phosphorylation levels of p44 and p42 MAPK at Thr202 / Tyr204 was significantly higher in overloaded LZ and OZ muscles at both 7 and 21 days $(P<0.05$; Fig. 4). The magnitude of ERK1/2 phosphorylation was higher in LZ compared to OZ rats. The basal phosphorylation levels of p42 MAPK were 


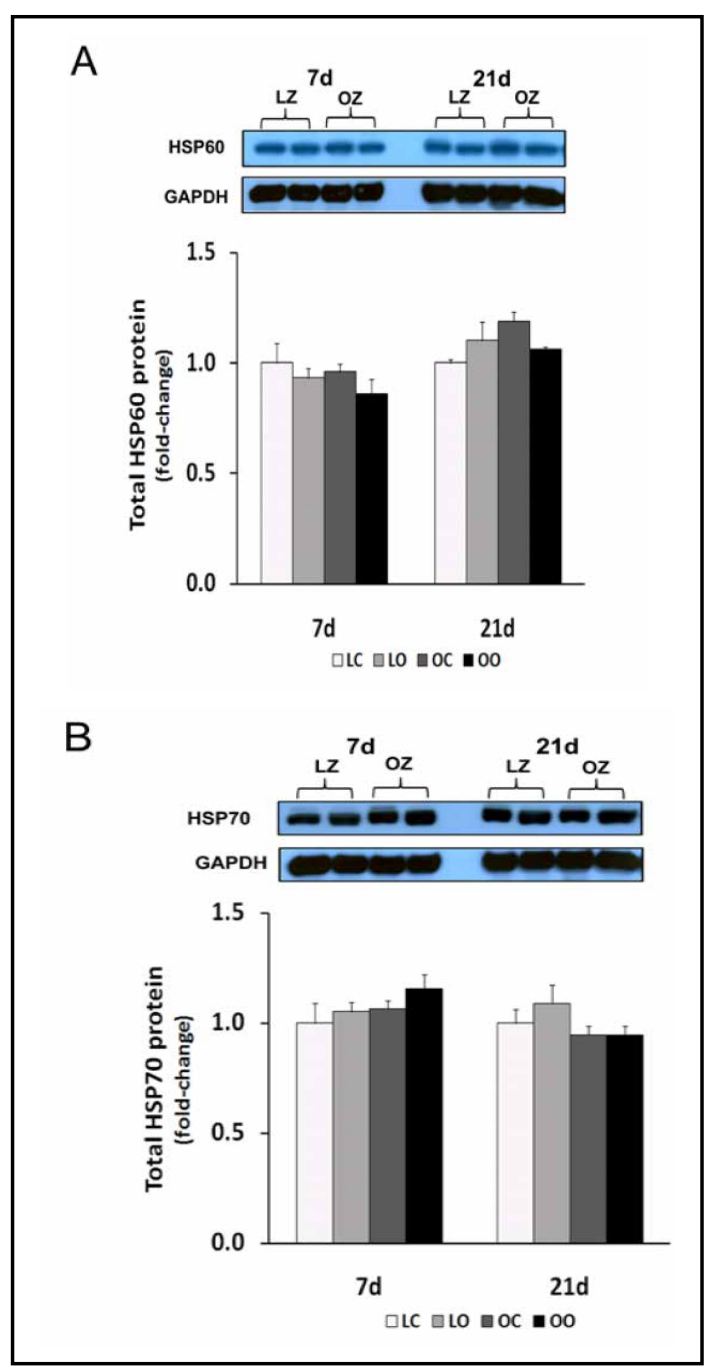

Fig. 2. Immunoblot analysis of the expression levels of heat shock proteins,(A) HSP60 and (B) HSP70 in control and overloaded soleus muscles of LZ and OZ. Protein quantification normalized to the abundance of GAPDH protein; $\mathrm{n}=6$.
A

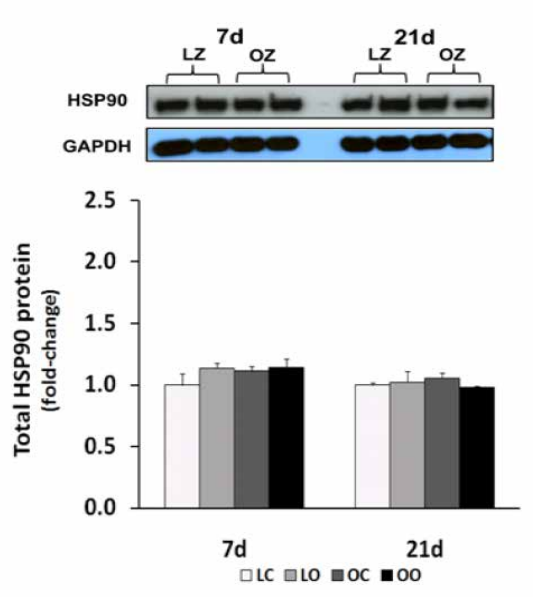

B

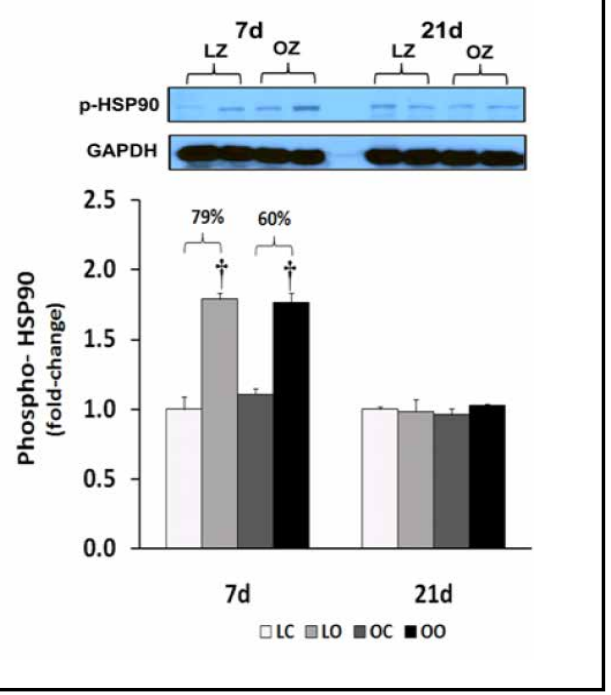

Fig. 3. Immunoblot analysis of the heat shock protein, HSP90 (A) protein content and (B) phosphorylation at Thr5/7 in control and loaded soleus muscles of LZ and OZ. Protein quantification normalized to the abundance of GAPDH protein; $n=6$. †Significantly different from contralateral control muscle, $P<0.05$.

significantly higher in insulin resistant soleus muscle compared to normal soleus muscle $(P<0.05$; Fig. 4). Muscle overload did not alter the expression of JNK/SAPK in either the LZ or OZ solei muscles (Fig. 5). The phosphorylation level of p46 (JNK1) at Thr183/Tyr185 was significantly higher in both LZ and OZ rat with overload at both 7 and 21 days, while the degree of phosphorylation was higher in the LZ compared to OZ soleus $(P<0.05$; Fig. 4).

Muscle specific miRNA expression following muscle overload is influenced by insulin resistance

The amount of mir-1 and miR133 in insulin resistant soleus muscles was less than that observed in the normal soleus muscle ( $P<0.05$; Fig. 6 and 7). Both 7 and 21 days overload decreased mir-1 levels in the LZ and OZ soleus muscles, but the magnitude of decrease was higher in normal muscle when compared to the insulin-resistant muscle $(\mathrm{P}<0.05$; Fig. 6). 
A
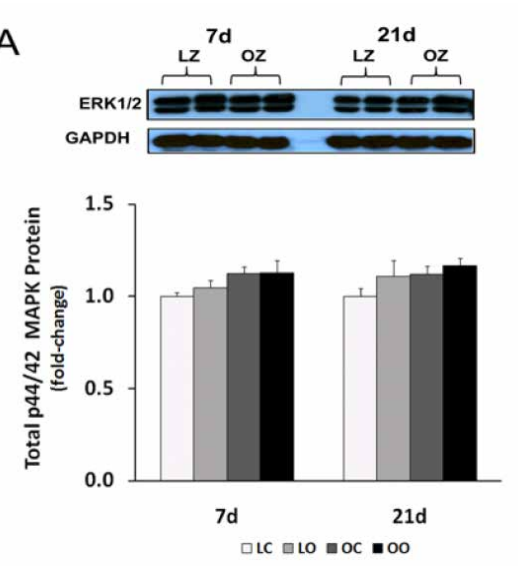

B
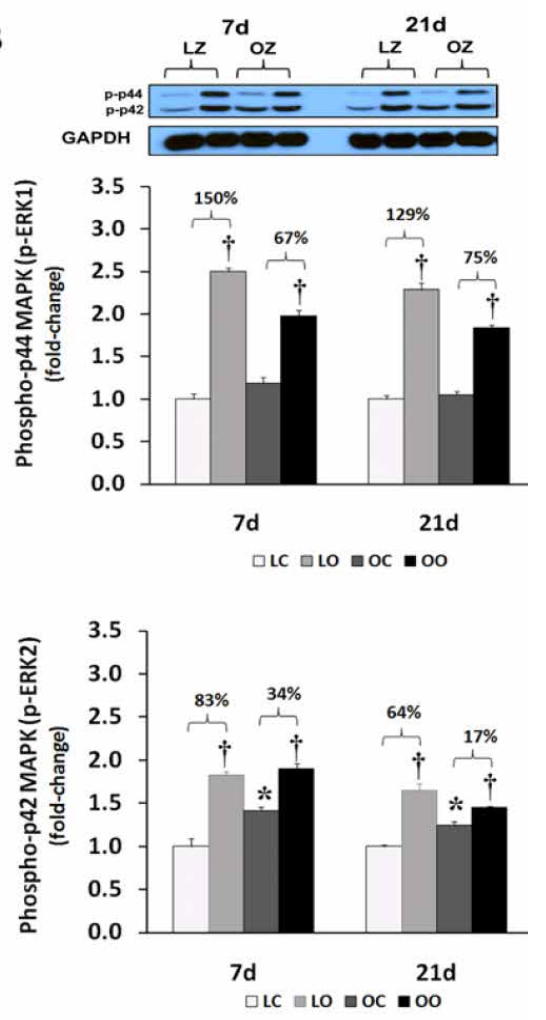

Fig. 4. Immunoblot analysis of the p44/42(ERK1/2) (A) protein content and (B) phosphorylation at Thr202/Tyr204 in control and loaded soleus muscles of LZ and OZ. Protein quantification normalized to the abundance of GAPDH; $n=6$. $\dagger$ Significantly different from contralateral control muscle, *Significantly different from lean control value, $P<0.05$.

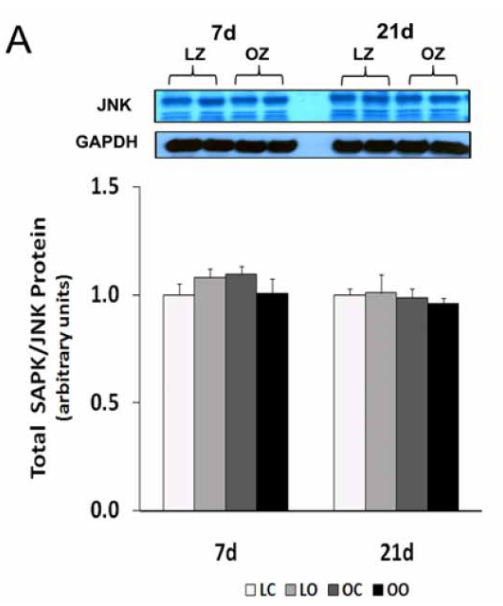

B
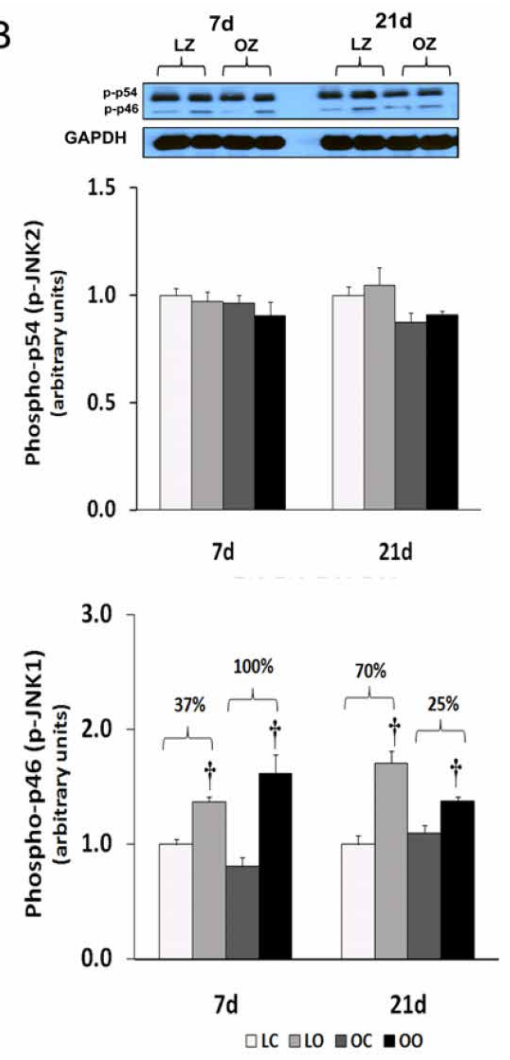

Fig. 5. Immunoblot analysis of the SAPK/JNK (A) protein content and (B) phosphorylation at Thr183/ Tyr185 in control and loaded soleus muscles of LZ and OZ. Protein quantification normalized to the abundance of GAPDH protein; $n=6$. †Significantly ifferent from contralateral control muscle, $P<0.05$.

The expression levels of miR-133a decreased significantly in the LZ animals with overload at both 7 and 21 days $(\mathrm{P}<0.05$; Fig. 7). In contrast, miR-133a levels in the $0 \mathrm{Z}$ rat were increased significantly with overload at both 7 and 21 days ( $<<0.05$; Fig. 7 ). 


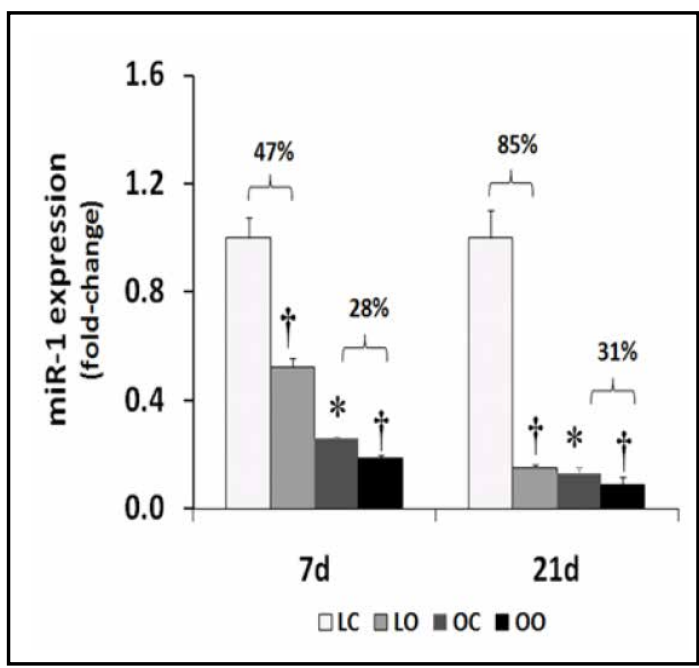

Fig. 6. Insulin resistance alters the regulation of miR-1 with overload. Quantitative RT-PCR analyses of miR-1 expression in control and loaded soleus muscles of $\mathrm{LZ}$ and $\mathrm{OZ}$ rats. $n=6$. †Significantly different from contralateral control muscle, *Significantly different from lean control value, $P<0.05$.

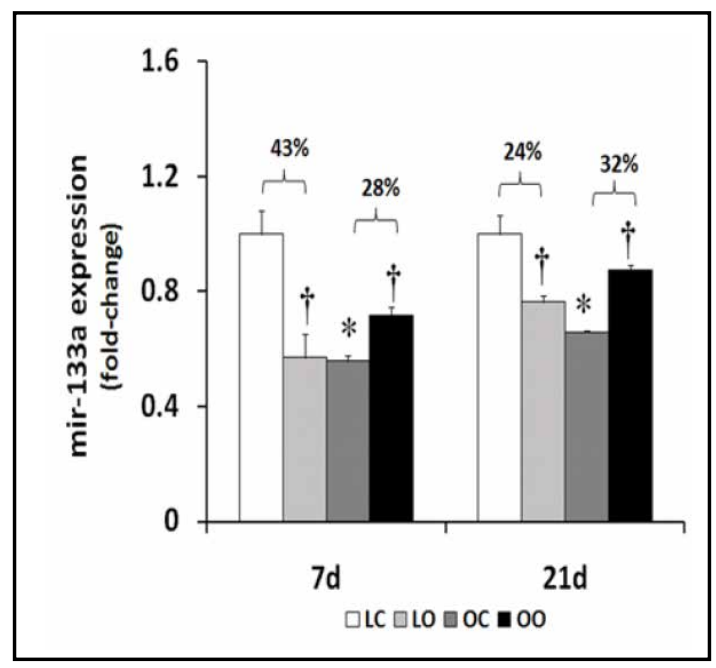

Fig. 7. Insulin resistance alters the regulation of miR-133a with overload. Quantitative RT-PCR analyses of miR-133a expression in control and loaded soleus muscles of $\mathrm{LZ}$ and $\mathrm{OZ}$ rats. $n=6$. †Significantly different from contralateral control muscle, *Significantly different from lean control value, $P<0.05$.

\section{Discussion}

Skeletal muscle adaptation to increased load is characterized by increases in muscle mass (hypertrophy) that are thought to be regulated by the activation of several signaling cascades. Previously we demonstrated that the degree of muscle hypertrophy following an overload stimulus was decreased in the insulin-resistant obese Zucker ( $\mathrm{fa} / \mathrm{fa}$ ) rat (OZ) when compared to that observed in the lean Zucker rat (LZ) and that this attenuation was characterized by decrements in mammalian target of rapamycin (mTOR) and its downstream translational signaling intermediates, p70S6k, rpS6 and eukaryotic initiation factor 4E-binding protein 1 (4E-BP1) [1]. Here we extend upon those findings and demonstrate overload in the $\mathrm{OZ}$ rat soleus is associated with diminished HSP27 phosphorylation, decreased MAPK phosphorylation and alterations in the regulation of muscle specific miRNAs.

Insulin resistance is associated with alterations in HSP27 phosphorylation following muscle overload

Previous reports have demonstrated that HSP27 expression increases in response to increased muscle load in both human and animal muscles $[8,37,38]$. Consistent with these data, here we show that HSP27 expression was increased with overload in both the $\mathrm{LZ}$ and $\mathrm{OZ}$ soleus muscles. Overload also led to a significant increase in the phosphorylation or activation of HSP27 at Ser82 in normal muscle; however this response appeared to be attenuated in the insulin-resistant soleus. In vitro studies have suggested thought that the increased phosphorylation of HSP27 is involved in maintaining cytoskeletal protein integrity. Whether HSP27 exhibits a similar function in vivo is currently unclear and beyond the scope of the present study. Additionally, it is also unknown why insulin resistance was associated with diminished overload-induced phosphorylation of HSP27. Nonetheless, given that HSP27 is a substrate for the MAPK proteins [21, 39, 40], it is possible that the decreased activation of MAPK with overload seen in the OZ soleus (Fig. 4 \& 5) may be related. Future experiments designed to directly test this possibility will be required to definitely shed light in establish cause and effect. 
Insulin resistance is associated with decreases in overload-induced MAPK phosphorylation

A review of literature suggests that the MAPK proteins play an integral role in the adaptation of skeletal muscle to alterations in contractile loading [21, 41-44]. It is known that the activation of MAPK proteins is important in regulating several processes, including glucose uptake, cell replication, and protein synthesis $[45,46]$. The basal phosphorylation levels of p42- MAPK in the insulin-resistant soleus muscle were significantly higher compared to that observed in the normal soleus. Overload was found to increase the activation of p44- and p42-MAPK in both normal and insulin resistant soleus, however the magnitude of activation was higher in normal muscle compared to that observed in insulin resistant soleus. Given the possibility that ERK1/2 activation is associated with skeletal muscle hypertrophy [47, 48], the differences in magnitude of activation of ERK1/2 between lean and obese rats with overload may help to explain the decreased hypertrophic response seen in the $\mathrm{OZ}$ animals. Although the exact mechanism for ERK1/2 involvement in muscle hypertrophy has yet to be elucidated, it has been hypothesized that ERK1/2 activation is associated with elevations in translational initiation in a process involving increased phosphorylation of the eukaryotic initiation factor eIF4E [49].

\section{Muscle-specific miRNAs and Insulin-resistance}

It has been suggested that the expressions of mir-1, miR133 are decreased in response to functional overload [24], chronic endurance training [26], and resistance exercise [50]. Here we investigated whether insulin-resistance affects the expression and regulation of muscle-specific miRNAs with overload. Our semi-quantitative PCR data demonstrated that insulin resistance was associated with a lower expression of mir-1 and mir-133 compared to that observed in the non-diabetic soleus. The physiological significance of diminshed muscle-specific miRNAs with insulin resistance are currently unclear. However, recent data suggest that the expression of mir-1 and mir-133, at least in C2C12 cells, may be regulated by the myogenic regulatory factors (MRFs) myogenin and MyoD [51]. Whether a similar finding exists in vivo is currently unclear. Previous work from our laboratory failed to demonstrate differences in the regulation of MRFs with overload in OZ rat [1]. These findings are consistent with the possibility that these MRFs may play a diminished role in regulating the expression of mir-1 and mir-133 in LZ and OZ animals. Similarly, like that observed for most of the known miRNA species, the exact role that these molecules may play in vivo has yet to be fully elucidated.

In addition to the possibility that insulin resistance may affect miRNA expression profiles, we also observed that muscle overload is associated diminished mir-1 expression in both the LZ and OZ rats (Fig. 6). These results are in agreement with recent findings by McCarthy and Esser, who reported that 50\% decrease in expression of mir- 1 after 7 -days functional overload of the mouse plantaris muscle [24]. Interestingly, the overload associated decreases observed in the present study appeared to be higher in the LZ compared to $\mathrm{OZ}$ after 7- and 21-days of overload (Fig. 6). Similar results to what we had observed with mir-1 were seen when we examined mir-133a expression in the LZ animals (Fig. 7). Conversely, in the $\mathrm{OZ}$ animals, overload appeared to increase the expression of this molecule (Fig. 7). Whether these findings are directly related to the diminished overload-induced hypertrophy we see in the $\mathrm{OZ}$ rat soleus is currently unclear and beyond the scope of the present study. Similarly, why insulin-resistance might be associated with differences in how overload regulates miRNA expression is not known.

\section{Summary and conclusions}

The findings of the present study suggest that the impaired hypertrophic response seen in insulin resistant muscle appears to be associated with alterations in HSP and MAPK phosphorylation along with changes in the way muscle overload regulates muscle specific miRNA expression. These data extend our previous work showing that insulin resistance is characterized by differences in the overload induced activation of mTOR signaling. Nonetheless, it should be noted that additional studies are needed to better understand 
why differences might exist in the adaptation of insulin resistant skeletal muscle to overload stimuli. One potential area to investigate would be to look at effect of insulin resistance on the load-induced changes in growth factor and cytokine expression given that previous data has suggested that these molecules could be involved in regulating the phosphorylation of IRS-1 and insulin dependent signaling $[52,53]$. Similarly, how insulin resistance and overload might affect the amount and phosphorylation status of glycogen synthase kinase $\beta$, or other signaling molecules within the Akt signaling axis may also be informative. Future studies perhaps using pharmacological and genetic manipulation will be useful in assessing the cause and effect of our findings and may offer therapeutic insight for the maximization of muscle growth in diabetic individuals.

\section{Acknowledgements}

This work was funded in part through grant support from the Department of Energy, \#DE-PS02-09ER-01 to E. R. B.

\section{References}

1 Arvapalli RK, Paturi S, Laurino JP, Katta A, Kakarla SK, Gadde MK, Wu M, Rice KM, Walker EM, Wehner P, Blough ER: Deferasirox decreases age-associated iron accumulation in the aging f344xbn rat heart and liver. Cardiovasc Toxicol 2010;10:108-116.

- Paturi S, Gutta AK, Kakarla SK, Katta A, Arnold EC, Wu M, Rice KM, Blough ER: Impaired overload-induced hypertrophy in obese zucker rat slow-twitch skeletal muscle. J Appl Physiol 2010;108:7-13.

- 3 Tupling AR, Bombardier E, Vigna C, Quadrilatero J, Fu M: Interaction between hsp70 and the sr ca ${ }^{2+}$ pump: A potential mechanism for cytoprotection in heart and skeletal muscle. Appl Physiol Nutr Metab 2008;33:1023-1032.

-4 Nelson RJ, Ziegelhoffer T, Nicolet C, Werner-Washburne M, Craig EA: The translation machinery and $70 \mathrm{kd}$ heat shock protein cooperate in protein synthesis. Cell 1992;71:97-105.

5 Henics T, Nagy E, Oh HJ, Csermely P, von Gabain A, Subjeck JR: Mammalian hsp70 and hsp110 proteins bind to rna motifs involved in mrna stability. J Biol Chem 1999;274:17318-17324.

6 Kamradt MC, Chen F, Sam S, Cryns VL: The small heat shock protein alpha b-crystallin negatively regulates apoptosis during myogenic differentiation by inhibiting caspase-3 activation. J Biol Chem 2002;277:3873138736.

7 Sakurai T, Fujita Y, Ohto E, Oguro A, Atomi Y: The decrease of the cytoskeleton tubulin follows the decrease of the associating molecular chaperone alphab-crystallin in unloaded soleus muscle atrophy without stretch. FASEB J 2005;19:1199-1201.

8 Huey KA: Regulation of hsp25 expression and phosphorylation in functionally overloaded rat plantaris and soleus muscles. J Appl Physiol 2006;100:451-456.

-9 Huey KA, Burdette S, Zhong H, Roy RR: Early response of heat shock proteins to functional overload of the soleus and plantaris in rats and mice. Exp Physiol 2010;95:1145-1155.

10 Locke M: Heat shock protein accumulation and heat shock transcription factor activation in rat skeletal muscle during compensatory hypertrophy. Acta Physiol (Oxf) 2008;192:403-411.

-11 Bodine SC, Stitt TN, Gonzalez M, Kline WO, Stover GL, Bauerlein R, Zlotchenko E, Scrimgeour A, Lawrence JC, Glass DJ, Yancopoulos GD: Akt/mtor pathway is a crucial regulator of skeletal muscle hypertrophy and can prevent muscle atrophy in vivo. Nature cell biology 2001;3:1014-1019.

12 Rommel C, Bodine SC, Clarke BA, Rossman R, Nunez L, Stitt TN, Yancopoulos GD, Glass DJ: Mediation of igf1-induced skeletal myotube hypertrophy by pi(3)k/akt/mtor and pi(3)k/akt/gsk3 pathways. Nature cell biology 2001;3:1009-1013.

13 Baar K, Esser K: Phosphorylation of p70(s6k) correlates with increased skeletal muscle mass following resistance exercise. Am J Physiol 1999;276:C120-127. 
Katta/Thulluri/Manne et al.: Alterations in HSPs and miRNA in Insulin-resistant Skeletal Muscle Subjected to Overload

14 Sherwood DJ, Dufresne SD, Markuns JF, Cheatham B, Moller DE, Aronson D, Goodyear LJ: Differential regulation of map kinase, p70(s6k), and akt by contraction and insulin in rat skeletal muscle. Am J Physiol 1999;276:E870-878.

15 Widmann C, Gibson S, Jarpe MB, Johnson GL: Mitogen-activated protein kinase: Conservation of a threekinase module from yeast to human. Physiol Rev 1999;79:143-180.

16 Shi H, Scheffler JM, Zeng C, Pleitner JM, Hannon KM, Grant AL, Gerrard DE: Mitogen-activated protein kinase signaling is necessary for the maintenance of skeletal muscle mass. Am J Physiol Cell Physiol 2009;296:C1040-1048.

17 Canagarajah BJ, Khokhlatchev A, Cobb MH, Goldsmith EJ: Activation mechanism of the map kinase erk2 by dual phosphorylation. Cell 1997;90:859-869.

18 Roux PP, Blenis J: Erk and p38 mapk-activated protein kinases: A family of protein kinases with diverse biological functions. Microbiol Mol Biol Rev 2004;68:320-344.

19 Fluckey JD, Knox M, Smith L, Dupont-Versteegden EE, Gaddy D, Tesch PA, Peterson CA: Insulin-facilitated increase of muscle protein synthesis after resistance exercise involves a map kinase pathway. Am J Physiol Endocrinol Metab 2006;290:E1205-1211.

20 Carlson CJ, Fan Z, Gordon SE, Booth FW: Time course of the mapk and pi3-kinase response within 24 h of skeletal muscle overload. J Appl Physiol 2001;91:2079-2087.

21 Thompson HS, Maynard EB, Morales ER, Scordilis SP: Exercise-induced hsp27, hsp70 and mapk responses in human skeletal muscle. Acta Physiol Scand 2003;178:61-72.

22 Kramer HF, Goodyear LJ: Exercise, mapk, and nf-kappab signaling in skeletal muscle. J Appl Physiol 2007;103:388-395.

23 Boppart MD, Aronson D, Gibson L, Roubenoff R, Abad LW, Bean J, Goodyear LJ, Fielding RA: Eccentric exercise markedly increases c-jun nh(2)-terminal kinase activity in human skeletal muscle. J Appl Physiol 1999;87:1668-1673.

24 McCarthy JJ, Esser KA: Microrna-1 and microrna-133a expression are decreased during skeletal muscle hypertrophy. J Appl Physiol 2007;102:306-313.

25 Roth SM: MicroRNAs: Playing a big role in explaining skeletal muscle adaptation? J Appl Physiol 2011;110:301-2.

26 Nielsen S, Scheele C, Yfanti C, Akerstrom T, Nielsen AR, Pedersen BK, Laye M: Muscle specific micrornas are regulated by endurance exercise in human skeletal muscle. J Physiol 2010;588:4029-4037.

27 Ge Y, Chen J: Micrornas in skeletal myogenesis. Cell Cycle 2011;10:441-8.

28 Cannell IG, Kong YW, Bushell M: How do micrornas regulate gene expression? Biochem Soc Trans 2008;36:1224-1231.

29 Xu C, Lu Y, Pan Z, Chu W, Luo X, Lin H, Xiao J, Shan H, Wang Z, Yang B: The muscle-specific micrornas mir-1 and mir-133 produce opposing effects on apoptosis by targeting hsp60, hsp70 and caspase-9 in cardiomyocytes. J Cell Sci 2007;120:3045-3052.

-30 Sokol NS, Ambros V: Mesodermally expressed drosophila microrna-1 is regulated by twist and is required in muscles during larval growth. Genes Dev 2005;19:2343-2354.

-31 Chen JF, Mandel EM, Thomson JM, Wu Q Callis TE, Hammond SM, Conlon FL, Wang DZ: The role of microrna-1 and microrna-133 in skeletal muscle proliferation and differentiation. Nat Genet 2006;38:228233.

32 Kim HK, Lee YS, Sivaprasad U, Malhotra A, Dutta A: Muscle-specific microrna mir-206 promotes muscle differentiation. J Cell Biol 2006;174:677-687.

33 Blough ER, Linderman JK: Lack of skeletal muscle hypertrophy in very aged male fischer $344 \mathrm{x}$ brown norway rats. J Appl Physiol 2000;88:1265-1270.

-34 Katta A, Karkala SK, Wu M, Meduru S, Desai DH, Rice KM, Blough ER: Lean and obese zucker rats exhibit different patterns of p70s6 kinase regulation in the tibialis anterior muscle in response to high-force muscle contraction. Muscle Nerve 2009;39:503-511.

- 35 Katta A, Preston DL, Karkala SK, Asano S, Meduru S, Mupparaju SP, Yokochi E, Rice KM, Desai DH, Blough ER: Diabetes alters contraction-induced mitogen activated protein kinase activation in the rat soleus and plantaris. Exp Diabetes Res 2008;2008:738101.

-36 Livak KJ, Schmittgen TD: Analysis of relative gene expression data using real-time quantitative pcr and the 2(-delta delta c(t)) method. Methods 2001;25:402-408. 
Katta/Thulluri/Manne et al.: Alterations in HSPs and miRNA in Insulin-resistant Skeletal Muscle Subjected to Overload

37 Naito H, Powers SK, Demirel HA, Sugiura T, Dodd SL, Aoki J: Heat stress attenuates skeletal muscle atrophy in hindlimb-unweighted rats. J Appl Physiol 2000;88:359-363.

- 38 Oishi Y, Ishihara A, Talmadge RJ, Ohira Y, Taniguchi K, Matsumoto H, Roy RR, Edgerton VR: Expression of heat shock protein 72 in atrophied rat skeletal muscles. Acta Physiol Scand 2001;172:123-130.

39 Dorion S, Landry J: Activation of the mitogen-activated protein kinase pathways by heat shock. Cell Stress Chaperones 2002;7:200-206.

40 Keller JM, Escara-Wilke JF, Keller ET: Heat stress-induced heat shock protein 70 expression is dependent on erk activation in zebrafish (danio rerio) cells. Comp Biochem Physiol A Mol Integr Physiol 2008;150:307314.

-41 Benziane B, Burton TJ, Scanlan B, Galuska D, Canny BJ, Chibalin AV, Zierath JR, Stepto NK: Divergent cell signaling after short-term intensified endurance training in human skeletal muscle. Am J Physiol Endocrinol Metab 2008;295:E1427-1438.

42 Hawley JA, Hargreaves M, Zierath JR: Signalling mechanisms in skeletal muscle: Role in substrate selection and muscle adaptation. Essays Biochem 2006;42:1-12.

43 Williamson D, Gallagher P, Harber M, Hollon C, Trappe S: Mitogen-activated protein kinase (mapk) pathway activation: Effects of age and acute exercise on human skeletal muscle. J Physiol 2003;547:977-987.

-44 Wretman C, Widegren U, Lionikas A, Westerblad H, Henriksson J: Differential activation of mitogenactivated protein kinase signalling pathways by isometric contractions in isolated slow- and fast-twitch rat skeletal muscle. Acta Physiol Scand 2000;170:45-49.

45 Sakamoto K, Goodyear LJ: Invited review: Intracellular signaling in contracting skeletal muscle. J Appl Physiol 2002;93:369-383.

-46 Widegren U, Ryder JW, Zierath JR: Mitogen-activated protein kinase signal transduction in skeletal muscle: Effects of exercise and muscle contraction. Acta Physiol Scand 2001;172:227-238.

-47 Haddad F, Adams GR: Inhibition of map/erk kinase prevents igf-i-induced hypertrophy in rat muscles. J Appl Physiol 2004;96:203-210.

-48 Shi H, Zeng C, Ricome A, Hannon KM, Grant AL, Gerrard DE: Extracellular signal-regulated kinase pathway is differentially involved in beta-agonist-induced hypertrophy in slow and fast muscles. Am J Physiol Cell Physiol 2007;292:C1681-1689.

49 Wang X, Flynn A, Waskiewicz AJ, Webb BL, Vries RG, Baines IA, Cooper JA, Proud CG: The phosphorylation of eukaryotic initiation factor eif4e in response to phorbol esters, cell stresses, and cytokines is mediated by distinct map kinase pathways. J Biol Chem 1998;273:9373-9377.

-50 Drummond MJ, McCarthy JJ, Fry CS, Esser KA, Rasmussen BB: Aging differentially affects human skeletal muscle microrna expression at rest and after an anabolic stimulus of resistance exercise and essential amino acids. Am J Physiol Endocrinol Metab 2008;295:E1333-1340.

51 Rao PK, Kumar RM, Farkhondeh M, Baskerville S, Lodish HF: Myogenic factors that regulate expression of muscle-specific micrornas. Proc Natl Acad Sci U S A 2006;103:8721-8726.

52 Adams GR, Haddad F: The relationships among igf-1, DNA content, and protein accumulation during skeletal muscle hypertrophy. J Appl Physiol 1996;81:2509-2516.

53 Wei Y, Chen K, Whaley-Connell AT, Stump CS, Ibdah JA, Sowers JR: Skeletal muscle insulin resistance: Role of inflammatory cytokines and reactive oxygen species. Am J Physiol Regul Integr Comp Physiol 2008;294:R673-680. 